\title{
Saddle points for maximin investment problems with observable but non-predictable parameters: solution via heat equation *
}

\author{
Nikolai Dokuchaev \\ Department of Mathematics and Statistics, University of Limerick, Ireland
}

\begin{abstract}
We study optimal investment problem for a market model where the evolution of risky assets prices is described by Itô's equations. The risk-free rate, the appreciation rates, and the volatility of the stocks are all random; they depend on a random parameter that is not adapted to the driving Brownian motion. The distribution of this parameter is unknown. The optimal investment problem is stated in a maximin setting to ensure that a strategy is found such that the minimum of expected utility over all possible distributions of parameters is maximal. We show that a saddle point exists and can be found via solution of the standard one-dimensional heat equation with a Cauchy condition defined via one-dimensional minimization. This solution even covers models with unknown solution for a given distribution of the market parameters.
\end{abstract}

Key words: continuous time market models, uncertainty, minimax problems, optimal portfolio, saddle point

Mathematical Subject Classification (1991): 49J35, 49K35, 60J70, 93E20

\section{Introduction}

This paper studies optimal investment problem for a diffusion market consisting of a finite number of risky assets (for example, bonds, stocks, or options). Risky assets evolution is

*IMA J. Management Mathematics 17 (2006) 257-276. 
described by Itô's equation. We assume also that there is a bank account where money grows exponentially according to the short rate (we shall call it risk-free rate).

The classical optimal investment problem is to find a portfolio strategy for which $\mathbf{E} U(X(T))$ is to be maximized, where $\mathbf{E}$ denotes the mathematical expectation, $U(\cdot)$ is an utility function, and where $X(T)$ represents the wealth at the final time $T$. There are many works devoted to different modifications of this problem (see, e.g., Merton (1969), and survey in Hakansson (1997) and Karatzas and Shreve (1998)). If the market parameters are assumed to satisfy an Itô's equation, then the solution of the optimal investment problem can be obtained via dynamic programming approach and solution of a Bellman equation (or a Hamilton-Jacobi-Bellman equation). This approach is working if the current vector of market parameters $[r(t), a(t), \sigma(t)]$ can be represented as $f(y(t))$ for some deterministic function $f(\cdot)$ and some random process $y(t)$, such that $[S(t), y(t)]$ is a Markov process. Here $S(t)=\left[S_{1}(t), \ldots, S_{n}(t)\right]$ is the vector of stock prices, $n$ is the number of different stocks, $r(t)$ is the risk-free interest rate, $a(t)$ is the vector of the appreciation rate (in the financial literature, $a(t)$ is also called the drift coefficient), $\sigma(t)$ is the volatility matrix. The Bellman equation is nonlinear and it is usually degenerate. Besides, the dimension $m$ of the Bellman equation can be high. Since $m$ is the dimension of the process $[S(t), y(t)]$, then $m \geq n$. This dimension can be large even for generic models: if $\sigma(t)=y(t)$ and this process is independent of the driving Wiener process, then $m \geq n+n^{2}$. If a Bellman equation is degenerate and has a high dimension, then it is not easy to find its solution and optimal strategies. Optimal strategies were obtained for several special cases, including the case of random volatility $\sigma(t)$ (Chacko and Viceira (2005), Fleming and HernàndezHernàndez (2003), Sircar and Zariphopoulou (2005)), the case of random appreciation rate $a(t)$ (Williams (1977), Detemple (1986), Dothan and Feldman (1986), Gennotte (1986), Brennan (1998)), and the case of random risk free rate $r(t)$ (Fleming and Pang (2004), Munk and Sorensen (2004)). In any case, the Bellman equation has a Cauchy boundary condition at terminal time $T$, and its coefficients include the coefficients of the Itô's equation for $y(\cdot)$ (i.e., the equation for the market parameters). This means that if we want to find the solution of the Bellman equation at time $t$, then we need to know the distributions of the market parameters for time period $(t, T]$ (i.e., the future distributions). Therefore, this approach need to be modified for a case of uncertainty in prior distributions.

Another approach is based on martingale representation and Malliavin calculus. Using 
this method, Lakner (1995), (1998) found the martingale representation of the optimal portfolio, which is explicit in terms of a conditional expectation of a Malliavin derivative when $a(\cdot)$ is a non-observable Ornstein-Uhlenbeck process, and when $r(\cdot)$ and $\sigma(\cdot)$ are deterministic. Using the martingale approach, Sarr and Haussmann (2004) found the optimal portfolio for $a(\cdot)$ driven by a non-observable continuous Markov chain. Cvitanic et al (2005) found an explicit solution for the case when $a$ is a non-observable and Gaussian, and the utility is a power function. Similarly to the Bellman equation, the martingale approach also requires future distributions of the parameters, since their evolution law is assumed to be known.

For problems with uncertainty in prior distributions, the most popular and straightforward approach is solution of a maximization problem in maximin setting: Find a strategy which maximizes the infimum of expected utility over all admissible parameters from a given class. The maximin setting has long history in optimization and optimal control theory. It is presented in robust control, in particular, in $H^{p}$-control (see, e.g., the bibliography in Ackermann (1993) or Bryson (1996)). In economics, there is a large literature devoted to related investment problems. Uncertainty in prior probability measures is referred sometimes as the Knightian Uncertainty. Maximin setting in mathematical economics is presented in theory of problems with robust performance criteria. Investors with robust performance preferences choose their portfolios under the worst realization of the independent random variables. Dynamic portfolio selection problems with robust preferences have been considered by Gilboa and Schmeidler (1989), Epstein and Chen (2002), Cvitanić and Karatzas (1999), and Cvitanić (2000), Epstein and Miao (2003), Epstein and Schneider (2002), Trojani and Vanini (2002), and others (see bibliography in these papers or in Föllmer and Schied (2002)). A related problem with unknown beliefs were considered by Lazrak and Zapatero (2004) who described sets of market parameters that correspond to given optimal strategies.

We also consider the problem as a maximin problem: Find a strategy which maximizes the infimum of expected utility over all admissible $(r(\cdot), a(\cdot), \sigma(\cdot))$ from a given class.

We study a diffusion market model such that the risk free rate $r(t)$, the appreciation rate $a(t)$, and the volatility matrix $\sigma(t)$ are all random, with unknown prior distributions and evolution law. They are not adapted to the driving Brownian motion, and they are supposed to be currently observable (i.e., it is a case of "totally unhedgeable" coefficients, 
according to Karatzas and Shreve (1998), Chapter 6). We consider the case when the risk-free rate, the appreciation rates, and the volatility of the stocks are all random; they depend on a random parameter that is not adapted to the driving Brownian motion. It is the case of "totally unhedgeable" coefficients, according to Karatzas and Shreve (1998), Chapter 6). We assume that the (unknown) distribution of parameters is fairly arbitrary, i.e., we consider the most wide class of possible distributions. The appreciation rates and volatility are observable, but the optimal strategy given any prior distribution depends on this distributions and it is not myopic (see Example 5.1).

We study the maximin problem on the basis of duality method known in convex optimization. We show that the duality theorem holds and a saddle point exists under some non-restrictive conditions. Thus, the maximin problem which, as far as we know, cannot be solved directly, is reduced to a minimax problem. Furthermore, we found that the minimax problem requires minimization only over a single scalar parameter $R=\int_{0}^{T}|\theta(t)|^{2} d t$, where $\theta(t)$ is the risk premium process. In other words, the saddle point can be found via minimization of $R$ only. Note that this parameter does not depend on relative performance of different stocks, and it is defined by the entire set of stocks. Similar effect was found by Dokuchaev and Haussmann (2001) for an optimal portfolio compression problem. Using this effect, the original maximin problem is effectively solved; the optimal strategy is derived via solution of the standard one-dimensional heat equation when the diffusion coefficient is defined by the minimal $R$. That is a deterministic parabolic equation with known fundamental solution. This equation has a Cauchy condition defined via an one dimensional optimization problem where the utility function and the minimal $R$ are presented. The solution of the Cauchy problem can be expressed explicitly via integral with known kernel. We repeat that the heat equation is one dimensional, and the maximization is also one-dimensional, even for a case of a large number of stocks $n$.

The novelty of this result is that, we obtained explicitly (under certain conditions) the solution of the maximin problem even for a case when the solution is unknown for a given distribution of the random parameters (see, e.g., Example 5.1 below). In other words, the solution in maximin setting with unknown prior distributions appears to be easier than for the problem with given prior distribution.

It is interesting that, for the class of strategies based on historical prices (or with non-observable parameters), we have that the cumulative risk premium $R$ is not necessary 
minimal for the "worst" market parameter presented at the saddle point for the maximin problem (see Example 5.2).

Cvitanić and Karatzas (1999) and Cvitanić (2000) considered a related minimax and maximin problems of minimizing $\mathbf{E}\left(\xi_{1}-X(T)\right)^{+}$subject to $X(T) \geq \xi_{2}$, where $\xi_{1}$ and $\xi_{2}$ are given claims, for similar admissible strategies which allow direct observations of appreciation rates (adapted to the driving Brownian motion). We consider more general utility functions. Besides, the novelty of our result is that the problem is reduced to minimization of the scalar $R$ and solution of the heat equation. Dokuchaev and Teo (1999) obtained a duality theorem for a problem in maximin setting for another class of admissible strategies that use historical prices only.

The paper is organized as follows. In Section two we collect notation and definitions, and we set up the market. The optimal investment problem is stated in Section three. In Section four, the main result is presented. In Section five, we give some examples to illustrate that our problem setting is meaningful, and we discuss also the similar setting with admissible strategies that use observations of $(S, r)$ only. The proofs are given in the Appendix.

\section{Definitions and the market model}

We are given a standard probability space $(\Omega, \mathcal{F}, \mathbf{P})$, where $\Omega=\{\omega\}$ is a set of elementary events, $\mathcal{F}$ is a complete $\sigma$-algebra of events, and $\mathbf{P}$ is a probability measure that describes a prior probability distributions.

\subsection{Market model}

We consider a market model in a generalized Black-Scholes framework. We assume that the market consists of a risk free asset or bank account with price $B(t), t \geq 0$, and $n$ risky stocks with prices $S_{i}(t), t \geq 0, i=1,2, \ldots, n$, where $n<+\infty$ is given.

We assume that

$$
B(t)=B(0) \exp \left(\int_{0}^{t} r(s) d s\right)
$$

where and $r(t)$ is the random process of the risk-free interest rate (or the short rate). We assume that $B(0)=1$. The process $B(t)$ will be used as numeraire. 
The prices of the stocks evolve according to

$$
d S_{i}(t)=S_{i}(t)\left[a_{i}(t) d t+\sum_{j=1}^{n} \sigma_{i j}(t) d w_{j}(t)\right], \quad t>0,
$$

where $w(\cdot)=\left(w_{1}(\cdot), \ldots, w_{n}(\cdot)\right)$ is a standard Wiener process with independent components, $a_{i}(t)$ are the appreciation rates, and $\sigma_{i j}(t)$ are the volatility coefficients. The initial price $S_{i}(0)>0$ is a given non-random constant.

We assume that

We assume that $r(t), a(t) \triangleq\left\{a_{i}(t)\right\}_{i=1}^{n}$, and $\sigma(t) \triangleq\left\{\sigma_{i j}(t)\right\}_{i, j=1}^{n}$ are uniformly bounded, measurable random processes.

Set $\mu(t) \triangleq(r(t), \widetilde{a}(t), \sigma(t))$, where $\widetilde{a}(t) \triangleq a(t)-r(t) \mathbf{1}$ and $\mathbf{1} \triangleq\{1,1, \ldots, 1\}^{\top} \in \mathbf{R}^{n}$. The vector $\mu$ represents the vector of market parameters.

Let $\left\{\mathcal{F}_{t}^{\mu}\right\}_{t \geq 0}$ be the filtration generated by the process $(S(t), \mu(t))$ completed with the null sets of $\mathcal{F}$. Clearly, $\mathcal{F}_{t}^{\mu}$ coincides with the filtration generated by the processes $(w(t), \mu(t))$, and with the filtration generated by the processes $(\widetilde{S}(t), \mu(t))$, where

$$
\widetilde{S}(t)=\left[\widetilde{S}_{1}(t), \ldots, \widetilde{S}_{n}(t)\right] \triangleq \exp \left(-\int_{0}^{t} r(s) d s\right) S(t) .
$$

\subsection{Wealth and strategies}

Let $X_{0}>0$ be the initial wealth at time $t=0$, and let $X(t)$ be the wealth at time $t>0$, $X(0)=X_{0}$. We assume that

$$
X(t)=\pi_{0}(t)+\sum_{i=1}^{n} \pi_{i}(t)
$$

where the pair $\left(\pi_{0}(t), \pi(t)\right)$ describes the portfolio at time $t$. The process $\pi_{0}(t)$ is the investment in the bond, $\pi_{i}(t)$ is the investment in the $i$ th stock, $\pi(t)=\left(\pi_{1}(t), \ldots, \pi_{n}(t)\right)^{\top}$, $t \geq 0$.

The process $\tilde{X}(t) \triangleq \exp \left(-\int_{0}^{t} r(s) d s\right) X(t)$ is called the normalized wealth.

Let $\mathbf{S}(t) \triangleq \operatorname{diag}\left(S_{1}(t), \ldots, S_{n}(t)\right)$ and $\widetilde{\mathbf{S}}(t) \triangleq \operatorname{diag}\left(\widetilde{S}_{1}(t), \ldots, \widetilde{S}_{n}(t)\right)$ be diagonal matrices with the corresponding diagonal elements.

The portfolio is said to be self-financing, if

$$
d X(t)=\pi(t)^{\top} \mathbf{S}(t)^{-1} d S(t)+\pi_{0}(t) B(t)^{-1} d B(t)
$$


It follows that for such portfolios

$$
d \widetilde{X}(t)=\pi(t)^{\top} \mathbf{S}(t)^{-1} d \widetilde{S}(t)
$$

so $\pi$ alone suffices to specify the portfolio; it is called a self-financing strategy.

Let $\mathcal{G}_{t}$ be a filtration such that $\mathcal{G}_{t} \subseteq \mathcal{F}_{t}$. Let $\widetilde{\Sigma}\left(\mathcal{G}\right.$.) be the class of all $\mathcal{G}_{t}$-adapted processes $\pi(\cdot)=\left(\pi_{1}(\cdot), \ldots, \pi_{n}(\cdot)\right)$ such that $\mathbf{E} \int_{0}^{T}|\pi(t)|^{2} d t<+\infty$. We shall consider classes $\widetilde{\Sigma}(\mathcal{G}$.) as classes of admissible strategies.

For an Euclidean space $E$ we shall denote by $B([0, T] ; E)$ the set of bounded measurable functions $f(t):[0, T] \rightarrow E$. By the definitions of $\widetilde{\Sigma}\left(\mathcal{F}^{\mu}\right)$ and $\mathcal{F}_{t}^{\mu}$, any admissible selffinancing strategy is of the form

$$
\pi(t)=\Gamma\left(t,\left.[S(\cdot), \mu(\cdot)]\right|_{[0, t]}\right),
$$

where $\Gamma(\cdot)$ is a measurable function, $\Gamma(t, \cdot): C\left([0, t] ; \mathbf{R}^{n}\right) \times B\left([0, t] ; \mathbf{R} \times \mathbf{R}^{n} \times \mathbf{R}^{n \times n}\right) \rightarrow \mathbf{R}^{n}$, $t \geq 0$.

Clearly, the random processes $\pi(\cdot)$ with the same $\Gamma(\cdot)$ in $(2.6)$ may be different for different $\mu(\cdot)=(r(\cdot), \widetilde{a}(\cdot), \sigma(\cdot))$. Hence we also introduce strategies defined by $\Gamma(\cdot)$ : the function $\Gamma(\cdot)$ in $(2.6)$ is said to be a $H$-strategy (to emphasize that this strategy is defined as a function on the flow of the historical data).

Let the initial wealth $X(0)$ be fixed. For an admissible self-financing strategy $\pi(\cdot)$ such that $\pi(t)=\Gamma\left(t,\left.[S(\cdot), \mu(\cdot)]\right|_{[0, t]}\right)$, the process $(\pi(t), X(t))$ is uniquely defined by $\Gamma(\cdot)$ and $\mu(\cdot)=(r(\cdot), \widetilde{a}(\cdot), \sigma(\cdot))$ given $w(\cdot)$. We shall use the notation $X(t, \Gamma(\cdot), \mu(\cdot))$ and $\tilde{X}(t, \Gamma(\cdot), \mu(\cdot))$ to denote the corresponding total wealth and the normalized wealth. Furthermore, we shall use the notation $S(t)=S(t, \mu(\cdot))$ and $\widetilde{S}(t)=\widetilde{S}(t, \mu(\cdot))$ to emphasize that the stock price processes are different for different $\mu(\cdot)$.

\subsection{Class of uncertainties}

We describe now distributions of $\mu(\cdot)$ and what we suppose to know about them.

We assume that there exist a finite-dimensional Euclidean space $\bar{E}$ and a compact subset $\mathcal{T} \subset \bar{E}$. This set $\mathcal{T}=\{\alpha\}$ will represent the set of possible values of an unknown parameter.

Further, we assume that there exist a measurable function $M(\cdot)=\left(M_{r}(\cdot), M_{a}(\cdot), M_{\sigma}(\cdot)\right):[0, T] \times \mathcal{T} \times C\left([0, T] ; \mathbf{R}^{n}\right) \rightarrow \mathbf{R} \times \mathbf{R}^{n} \times \mathbf{R}^{n \times n}$ that is 
uniformly bounded and such that $M(t, \alpha, \xi)$ is continuous in $(\alpha, \xi) \in \mathcal{T} \times C\left([0, t] ; \mathbf{R}^{n}\right)$ for all $t$. Moreover, we assume that there exists a constant $C_{M}>0$ such that

$$
\begin{array}{r}
\left.\sup _{t \in[0, T]}\left|\xi_{i}(t) M_{a i}(t, \alpha, \xi(\cdot))\right|_{[0, t]}\right)-\eta_{i}(t) M_{a i}\left(t, \alpha,\left.\eta(\cdot)\right|_{[0, t]}\right)\left|\leq C_{M} \sup _{t \in[0, T]}\right| \xi(t)-\eta(t) \mid, \\
\left.\sup _{t \in[0, T]}\left|\xi_{i}(t) M_{\sigma i}(t, \alpha, \xi(\cdot))\right|_{[0, t]}\right)-\eta_{i}(t) M_{\sigma i}\left(t, \alpha,\left.\eta(\cdot)\right|_{[0, t]}\right)\left|\leq C_{M} \sup _{t \in[0, T]}\right| \xi(t)-\eta(t) \mid, \\
\forall \xi, \eta \in C\left([0, T] ; \mathbf{R}^{n}\right), \alpha \in \mathcal{T}, i=1, \ldots, n,
\end{array}
$$

where $\xi_{i}, \eta_{i}, M_{a i}$ are the $i$ th components of the vectors $\xi=\left\{\xi_{i}\right\}_{i=1}^{n}, \eta=\left\{\eta_{i}\right\}_{i=1}^{n}$, and $M_{a}=\left\{M_{a i}\right\}$, and where $M_{\sigma i}$ is the $i$ th row of the matrix $M_{\sigma}=\left\{M_{\sigma i j}\right\}_{i, j=1}^{n}$.

Under these assumptions, the solution of $(2.2)$ is well defined as the unique strong solution of Itô's equation with $\mu(t)=(r(t), \widetilde{a}(t), \sigma(t))=M\left(t, \alpha,\left.S(\cdot)\right|_{[0, t]}\right)$ for any $\alpha \in \mathcal{T}$. Let $S_{\alpha}(\cdot)$ denote the corresponding solution.

For $\alpha \in \mathcal{T}$, set

$$
\begin{aligned}
& \bar{M}_{r}(t, \alpha) \triangleq M_{r}\left(t, \alpha,\left.S_{\alpha}(\cdot)\right|_{[0, t]}\right), \\
& \bar{M}_{a}(t, \alpha) \triangleq M_{a}\left(t, \alpha,\left.S_{\alpha}(\cdot)\right|_{[0, t]}\right), \\
& \bar{M}_{\sigma}(t, \alpha) \triangleq M_{\sigma}\left(t, \alpha,\left.S_{\alpha}(\cdot)\right|_{[0, t]}\right) .
\end{aligned}
$$

Definition 2.1 Let $\mathcal{A}(\mathcal{T})$ be a set of all random processes $\mu^{\prime}(t)=\left(r^{\prime}(t), \widetilde{a}^{\prime}(t), \sigma^{\prime}(t)\right)$ such that there exists a random vector $\Theta^{\prime}: \Omega \rightarrow \mathcal{T}$ independent of $w(\cdot)$ and such that

$$
\left\{\begin{array}{l}
r^{\prime}(t) \equiv \bar{M}_{r}\left(t, \Theta^{\prime}\right) \\
\tilde{a}^{\prime}(t) \equiv \bar{M}_{a}\left(t, \Theta^{\prime}\right) \\
\sigma^{\prime}(t) \equiv \bar{M}_{\sigma}\left(t, \Theta^{\prime}\right) .
\end{array}\right.
$$

We assume that $\mu(\cdot) \in \mathcal{A}(\mathcal{T})$, and that is the only a prior information available.

Notice that the solution of $(2.2)$ is well defined for any $\mu(\cdot) \in \mathcal{A}(\mathcal{T})$, but the market is incomplete because of presence of the unhedgeable random factor $\Theta$.

In this setting, $\mathcal{T}$ is the set of possible values of the unknown parameter (random factor) $\Theta$; any $\alpha \in \mathcal{T}$ can be a value of $\Theta$.

In fact, the solution of investment problem obtained below does not require to know $\bar{E}, \mathcal{T}$, and $M(\cdot)$. It uses only a single scalar characteristic of $(\bar{E}, \mathcal{T}, M(\cdot))$ (see Corollary 4.1 below).

For $\alpha \in \mathcal{T}$, set

$$
\mu_{\alpha}(t) \triangleq\left(\bar{M}_{r}(t, \alpha), \bar{M}_{a}(t, \alpha), \bar{M}_{\sigma}(t, \alpha)\right)
$$

where $\bar{M}_{r}(t, \alpha), \bar{M}_{a}(t, \alpha)$ and $\bar{M}_{\sigma}(t, \alpha)$ are as in Definition 2.1 . 
We introduce a special class of admissible $H$-strategies that is defined by the class of uncertainties.

Definition 2.2 Let $\mathcal{C}$ be the class of all functions $\Gamma(t, \cdot): C\left([0, t] ; \mathbf{R}^{n}\right) \times B\left([0, t] ; \mathbf{R} \times \mathbf{R}^{n} \times\right.$ $\left.\mathbf{R}^{n \times n}\right) \rightarrow \mathbf{R}^{n}, t \geq 0$, such that the corresponding strategy $\pi(\cdot)$ defined by (2.6) belongs to $\widetilde{\Sigma}\left(\mathcal{F}^{\mu}\right)$ for any $\mu(\cdot)=(r(\cdot), \widetilde{a}(\cdot), \sigma(\cdot)) \in \mathcal{A}(\mathcal{T})$ and

$$
\sup _{\mu(\cdot)=\mu_{\alpha}(\cdot): \alpha \in \mathcal{T}} \mathbf{E} \int_{0}^{T}|\pi(t)|^{2} d t<\infty .
$$

A function $\Gamma(\cdot) \in \mathcal{C}$ is said to be an admissible $H$-strategy.

\section{Problem statement}

Let $T>0$ be a given time horizon, and let $X_{0}$ be a given initial wealth. Let $U(\cdot): \mathbf{R} \rightarrow$ $\mathbf{R} \cup\{-\infty\}$ be a given measurable function such that $\left|U\left(X_{0}\right)\right|<+\infty$. Let $\mathbf{D} \subset \mathbf{R}$ be a given convex set, $X_{0} \in \mathbf{D}$.

We may state our general problem as follows: Find an admissible $H$-strategy $\Gamma(\cdot) \in$ $\mathcal{C}$ and the corresponding self-financing strategy $\pi(\cdot) \in \widetilde{\Sigma}\left(\mathcal{F}^{\mu}\right)$ that solves the following optimization problem:

$$
\begin{array}{lll}
\text { Maximize } & \min _{\mu(\cdot) \in \mathcal{A}(\mathcal{T})} \mathbf{E} U(\tilde{X}(T, \Gamma(\cdot), \mu(\cdot))) \text { over } \quad \Gamma(\cdot) \in \mathcal{C} \\
\text { subject to }\left\{\begin{array}{l}
X(0, \Gamma(\cdot), \mu(\cdot))=X_{0}, \\
\tilde{X}(T, \Gamma(\cdot), \mu(\cdot)) \in \mathbf{D} \text { a.s. } \quad \forall \mu(\cdot) \in \mathcal{A}(\mathcal{T}) .
\end{array}\right.
\end{array}
$$

In this setting, $U$ represents the utility function, and $\mathbf{D}$ represents the constraints for the wealth. The problem is stated as a maximin problem: we want to ensure that the minimum of expected utility over all possible $\mu$ is maximal.

\section{Additional assumptions}

To proceed further, we assume that Conditions 3.1-3.5 remain in force throughout this paper.

Condition 3.1 The function $U(x): \mathbf{R} \rightarrow \mathbf{R}$ is either concave or convex in $x \in \mathbf{D}$, and there exist constants $c>0$ and $q \in(0,1]$ such that

$$
\left|U(x)-U\left(x_{1}\right)\right| \leq c\left(1+|x|+\left|x_{1}\right|\right)^{2-q}\left|x-x_{1}\right|^{q} \quad \forall x, x_{1} \in \mathbf{D} .
$$


Notice that Condition 3.1 is not restrictive if $\mathbf{D}$ is a bounded interval. This case is not excluded and it covers the goal achieving problem as well as any problem where large deviations must be avoided with probability 1 . Condition 3.3 is also satisfied, for example, if $U(x)=\ln x$ or $U(x)=x^{\delta}, \delta<1$, and $\mathbf{D} \subset[\varepsilon,+\infty)$, where $\varepsilon>0$.

For the sake of generality, we do not exclude the cases when $U$ is decreasing or convex, though these cases are not so important for economical models.

The following condition ensures that the market model is non-degenerate and arbitrage free.

Condition 3.2 For any $\mu(\cdot) \in \mathcal{A}(\mathcal{T}), \sigma(t) \sigma(t)^{\top} \geq c I_{n}$ for all $t$ a.s, where $c=c_{\mu}>0$ is a constants.

Let

$$
\theta_{\mu}(t) \triangleq \sigma(t)^{-1} \widetilde{a}(t)
$$

be the risk premium process given $\mu$. Set

$$
R_{\mu} \triangleq \int_{0}^{T}\left|\theta_{\mu}(t)\right|^{2} d t
$$

Further, set

$$
\mathcal{Z}(t, \mu(\cdot)) \triangleq \exp \left(\int_{0}^{t} \theta_{\mu}(s)^{\top} d w(s)+\frac{1}{2} \int_{0}^{t}\left|\theta_{\mu}(s)\right|^{2} d s\right) .
$$

Condition 3.2 and boundedness of the function $M(\cdot)$ imply that $\mathbf{E} \exp \left(\frac{1}{2} R_{\mu_{\alpha}}\right)<+\infty$ for any $\alpha \in \mathcal{T}$, i.e., the Novikov condition is satisfied, and $\mathbf{E} \mathcal{Z}\left(T, \mu_{\alpha}(\cdot)\right)^{-1}=1$ for all $\alpha \in \mathcal{T}$.

For any $\alpha \in \mathcal{T}$, define a probability measure $\mathbf{P}_{*}^{\alpha}$ by

$$
\frac{d \mathbf{P}_{*}^{\alpha}}{d \mathbf{P}}=\mathcal{Z}\left(T, \mu_{\alpha}(\cdot)\right)^{-1} .
$$

Let $\mathbf{E}_{*}^{\alpha}$ be the corresponding expectation. By Girsanov Theorem, the process $\widetilde{S}\left(t, \mu_{\alpha}(\cdot)\right)$ is a martingale under $\mathbf{P}_{*}^{\alpha}$, i.e., this is an equivalent risk-neutral measure on the conditional probability space under the condition that $\Theta=\alpha$.

Let us discuss briefly some implications for our market model which we don't need for our proofs; however, they are useful for understanding the model. First, it can be shown that $\mathbf{P}_{*}^{\alpha}$ is an unique equivalent risk-neutral measure on the conditional probability space $(\Omega, \mathcal{F}, \mathbf{P}(\cdot \mid \Theta=\alpha))$, so the market is complete for this conditional probability space. Second, it follows that $\mathbf{E} \mathcal{Z}(T, \mu(\cdot))^{-1}=\mathbf{E E}\left\{\mathcal{Z}(T, \mu(\cdot))^{-1} \mid \Theta\right\}=1$ for any $\mu$ or $\Theta$, and 
that $\mathbf{P}_{*}^{\Theta}$ is a risk-neutral measure for the original market (and this measure depends on $\Theta$ and it is not unique). Therefore, our market model is arbitrage free but incomplete (in the general case).

The following condition requires solvability of a finite dimensional optimization problem for $U$.

Condition 3.3 There exists a measurable set $\Lambda \subseteq \mathbf{R}$, and a measurable function $F(\cdot, \cdot)$ : $(0, \infty) \times \Lambda \rightarrow \mathbf{D}$ such that for each $z>0, \widehat{x}=F(z, \lambda)$ is a solution of the optimization problem

$$
\text { Maximize } z U(x)-\lambda x \quad \text { over } x \in \mathbf{D} \text {. }
$$

Moreover, this solution is unique for a.e. $z>0$.

Notice that Condition 3.3 is easy to verify, since the optimization problem is scalar. Several examples of calculating $F$ can be found in Dokuchaev and Teo (1999), Dokuchaev and Haussmann (2001), and Dokuchaev and Zhou (2001).

The following condition imposes restrictions on the solution $F$ of problem (3.5).

Condition 3.4 For any $\alpha \in \mathcal{T}$, there exist $\widehat{\lambda}_{\alpha} \in \Lambda, C=C_{\alpha}>0$, and $c_{0}=c_{0, \alpha} \in$ $\left(0,1 /\left(2 R_{\mu_{\alpha}}\right)\right)$ such that $F(\cdot, \hat{\lambda})$ is piecewise continuous on $(0, \infty), F\left(\mathcal{Z}\left(T, \mu_{\alpha}(\cdot)\right), \hat{\lambda}_{\alpha}\right)$ is $\mathbf{P}_{*}^{\alpha}$-integrable, and

$$
\left\{\begin{array}{l}
\mathbf{E}_{*}^{\alpha} F\left(\mathcal{Z}\left(T, \mu_{\alpha}(\cdot)\right), \widehat{\lambda}_{\alpha}\right)=X_{0}, \\
\left|F\left(z, \widehat{\lambda}_{\alpha}\right)\right| \leq C z^{c_{0} \log z} \quad \forall z>0 .
\end{array}\right.
$$

Examples when the imposed above condition is satisfied can be found in Dokuchaev and Haussmann (2001).

The following condition is restrictive (see, e.g., Example 5.2 below), however, it is essential for our approach.

Condition 3.5 There exists a non-random variable $R_{\min }$ such that there exists $\widehat{\alpha} \in \mathcal{T}$ such that $R_{\widehat{\mu}}=R_{\text {min }}$ and $R_{\widehat{\mu}} \leq R_{\mu}$ a.s. for all $\mu \in \mathcal{A}(\mathcal{T})$, where $\widehat{\mu} \triangleq \mu_{\widehat{\alpha}}$.

Clearly,

$$
R_{\text {min }}=\inf _{\mu(\cdot) \in \mathcal{A}(\mathcal{T})} R_{\mu}
$$

Remark 3.1 Condition 3.5 is satisfied if at least one of the following conditions holds: 
(i) $(\widetilde{a}(t), \sigma(t)) \equiv\left(M_{a}(t, \Theta), M_{\sigma}(t, \Theta)\right)$, where $\left(M_{a}(\cdot), M_{\sigma}(\cdot)\right):[0, T] \times \mathcal{T} \rightarrow \mathbf{R}^{n} \times \mathbf{R}^{n \times n}$ is a deterministic function ;

(ii) The matrix $\sigma(t)$ is diagonal, and

$$
\sigma(t) \equiv M_{\sigma}(t, \Theta), \quad \widetilde{a}_{i}(t)=\xi_{i}\left(t, \Theta,\left.S(\cdot)\right|_{[0, t]}\right) \eta_{i}(t, \Theta),
$$

where $M_{\sigma}(\cdot):[0, T] \times \mathcal{T} \rightarrow \mathbf{R}^{n \times n}, \xi_{i}(t, \cdot): \mathcal{T} \times C\left([0, t] ; \mathbf{R}^{n}\right) \rightarrow \mathbf{R}$ and $\eta_{i}(\cdot):$ $[0, T] \times \mathcal{T} \rightarrow \mathbf{R}$ are measurable functions such that $\left|\xi_{i}\left(t, \Theta,\left.S(\cdot)\right|_{[0, t]}\right)\right| \equiv 1, i=1, \ldots n$.

Remark 3.2 Our description of the class of admissible $\mu(\cdot)$ covers a setting when the minimum of $R_{\mu}$ over the class is given, or when the class of admissible $\mu(\cdot)$ is defined by a condition $R_{\mu} \in\left[R_{\min }, R_{\max }\right]$, where $R_{\text {min }}$ and $R_{\text {max }}$ are given, $0 \leq R_{\min }<R_{\max } \leq+\infty$. (It suffices to choose an appropriate pair $(\mathcal{T}, M(\cdot))$.)

\section{The main result: solution of the maximin problem}

We present the main result using the following steps. First, we derive the optimal strategy for for a fixed $\mu$ such that $\mu=\mu_{\alpha}$ for non-random $\alpha \in \mathcal{T}$ such that $\mathbf{R}_{\mu_{\alpha}}$ is deterministic. Using this result, we obtain the solution of the maximin problem with minimization over $\mu$ from the general class of random parameters.

\subsection{Preliminary result: optimal strategy for a fixed $\mu=\mu_{\alpha}$}

For given $R \geq 0, \lambda \in \Lambda, \mu(\cdot)$, let

$$
Y(t, \mu(\cdot)) \triangleq \int_{0}^{t} \theta_{\mu}(s)^{\top} \sigma(s)^{-1} \mathbf{S}(s)^{-1} d S(s), \quad \widehat{F}(y, R, \lambda) \triangleq F\left(e^{y+R / 2}, \lambda\right) .
$$

Let the function $u(\cdot)=u(\cdot, R, \lambda): \mathbf{R} \times[0, T] \rightarrow \mathbf{R}$ be the solution of the following Cauchy problem for the heat equation:

$$
\left\{\begin{array}{l}
\frac{\partial u}{\partial t}(x, t, R, \lambda)+\frac{R}{2 T} \frac{\partial^{2} u}{\partial x^{2}}(x, t, R, \lambda)=0 \\
u(x, T, R, \lambda)=\widehat{F}(x, R, \lambda)
\end{array}\right.
$$

where $F(\cdot)$ is defined in Condition 3.3 .

Further, for a given $\alpha \in \mathcal{T}$ such that $\mathbf{R}_{\mu_{\alpha}}$ is deterministic and $\mathbf{R}_{\mu_{\alpha}}>0$, let $H$-strategy $\widehat{\Gamma}_{\alpha}(\cdot)$ be defined as

$$
\widehat{\Gamma}_{\alpha}\left(t,\left.[S(\cdot), \mu(\cdot)]\right|_{[0, t]}\right) \triangleq B(t) \frac{\partial u}{\partial x}\left[Y(t, \mu(\cdot)), \tau_{\mu}\left(t, R_{\mu_{\alpha}}\right), R_{\mu_{\alpha}}, \lambda_{\alpha}\right] \widetilde{a}(t)^{\top} Q(t),
$$


where $\widehat{\lambda}_{\alpha}$ is defined from Condition 3.4, $Q(t) \triangleq\left(\sigma(t) \sigma(t)^{\top}\right)^{-1}$, and where

$$
\tau_{\mu}(t, R)=\tau\left(t,\left.[S(\cdot), \mu(\cdot)]\right|_{[0, t]}, R\right) \triangleq \frac{T}{R} \int_{0}^{t}\left|\theta_{\mu}(s)\right|^{2} d s .
$$

Note that Lemma 4.1(iii) below ensures that if $\mathbf{R}_{\mu_{\alpha}}$ is deterministic then $\widehat{\Gamma}_{\alpha}$ is uniquely defined even if $\lambda_{\alpha}$ is not unique.

Definition 4.1 Let $\mathcal{C}_{0}$ be the set of all admissible $H$-strategies $\Gamma(\cdot) \in \mathcal{C}$ such that

$$
\tilde{X}(T, \Gamma(\cdot), \mu(\cdot)) \in \mathbf{D} \quad \text { a.s. } \quad \forall \mu(\cdot) \in \mathcal{A}(\mathcal{T}) .
$$

We denote by $C^{2,1}(\mathbf{R} \times(0, T))$ the set of functions defined on $\mathbf{R} \times(0, T)$ which are continuous and have two continuous derivatives in the first variable and one in the second.

Lemma 4.1 (i) For any $R>0$ and $\lambda \in \Lambda$, problem (4.1) has the unique solution $u(\cdot, R, \lambda) \in C^{2,1}(\mathbf{R} \times(0, T))$ defined by (4.6), with $u(x, t, R, \lambda) \rightarrow \widehat{F}(x, R, \lambda)$ a.e. as $t \rightarrow T-0$.

(ii) For any $\alpha \in \mathcal{T}$ such that $R_{\mu_{\alpha}}>0$ is non-random, the strategy

$$
\begin{aligned}
\widehat{\pi}_{\alpha}(t)^{\top} & =\widehat{\Gamma}_{\alpha}\left(t,\left.\left[S(\cdot), \mu_{\alpha}(\cdot)\right]\right|_{[0, t]}\right)^{\top} \\
& =B(t) \frac{\partial u}{\partial x}\left(Y\left(t, \mu_{\alpha}(\cdot)\right), \tau_{\mu_{\alpha}}\left(t, R_{\mu_{\alpha}}\right), R_{\mu_{\alpha}}, \widehat{\lambda}_{\alpha}\right) \widetilde{a}(t)^{\top} Q(t)
\end{aligned}
$$

belongs to $\mathcal{C}_{0}$, the corresponding normalized wealth is

$$
\left.\widetilde{X}\left(t, \widehat{\Gamma}_{\alpha}(\cdot), \mu_{\alpha}(\cdot)\right)\right)=u\left(Y\left(t, \mu_{\alpha}(\cdot)\right), \tau_{\mu_{\alpha}}\left(t, R_{\mu_{\alpha}}\right), R_{\mu_{\alpha}}, \widehat{\lambda}_{\alpha}\right),
$$

and

$$
\mathbf{E} U\left(\widetilde{X}\left(T, \widehat{\Gamma}_{\alpha}(\cdot), \mu_{\alpha}(\cdot)\right)\right) \geq \mathbf{E} U\left(\tilde{X}\left(T, \Gamma(\cdot), \mu_{\alpha}(\cdot)\right)\right) \quad \forall \Gamma(\cdot) \in \mathcal{C}_{0} .
$$

(iii) The functions $F\left(\cdot, \widehat{\lambda}_{\alpha}\right), u\left(\cdot, R_{\mu_{\alpha}}, \widehat{\lambda}_{\alpha}\right), \widehat{\Gamma}_{\alpha}\left(\cdot, R_{\mu_{\alpha}}\right)$ as well as the probability distribution of the optimal normalized wealth $\tilde{X}\left(T, \widehat{\Gamma}_{\alpha}(\cdot), \mu_{\alpha}(\cdot)\right)$ is uniquely defined by $R_{\mu_{\alpha}}$ if $R_{\mu_{\alpha}}$ is non-random.

\subsection{Solution in maximin setting}

Now we are in the position to present the solution of problem (3.1)-(3.2)

$$
\begin{array}{lll}
\text { Maximize } & \min _{\mu(\cdot) \in \mathcal{A}(\mathcal{T})} \mathbf{E} U(\tilde{X}(T, \Gamma(\cdot), \mu(\cdot))) \text { over } \quad \Gamma(\cdot) \in \mathcal{C} \\
\text { subject to }\left\{\begin{array}{l}
X(0, \Gamma(\cdot), \mu(\cdot))=X_{0}, \\
\tilde{X}(T, \Gamma(\cdot), \mu(\cdot)) \in \mathbf{D} \text { a.s. } \quad \forall \mu(\cdot) \in \mathcal{A}(\mathcal{T}) .
\end{array}\right.
\end{array}
$$


By the definitions, this problem can be rewritten as

$$
\begin{array}{lc}
\text { Maximize } & \min _{\mu(\cdot) \in \mathcal{A}(\mathcal{T})} \mathbf{E} U(\tilde{X}(T, \Gamma(\cdot), \mu(\cdot))) \quad \text { over } \quad \Gamma(\cdot) \in \mathcal{C}_{0} \\
\text { subject to } & X(0, \Gamma(\cdot), \mu(\cdot))=X_{0}, \quad \forall \mu(\cdot) \in \mathcal{A}(\mathcal{T}) .
\end{array}
$$

Repeat that $R_{\text {min }}=\inf _{\mu(\cdot) \in \mathcal{A}(\mathcal{T})} R_{\mu}$.

Theorem 4.1 (i) If $R_{\min }=0$, then the trivial strategy, $\Gamma(\cdot) \equiv 0$, is the unique optimal strategy in $\mathcal{C}_{0}$ for problem (3.1)-(3.2).

(ii) Let $R_{\text {min }}>0$, and let $\widehat{\alpha} \in \mathcal{T}$ be such that $R_{\widehat{\mu}}=R_{\text {min }}$, where $\widehat{\mu} \triangleq \mu_{\widehat{\alpha}}$. Then the strategy

$$
\widehat{\pi}(t)^{\top}=\widehat{\Gamma}_{\widehat{\alpha}}\left(t,\left.[S(\cdot), \mu(\cdot)]\right|_{[0, t]}\right)
$$

belongs to $\mathcal{C}_{0}$ and is optimal in $\mathcal{C}_{0}$ for problem (3.1)-(3.2).

Remark 4.1 It follows from Theorem 4.1 and Theorem A.1 below that the point $\left(\widehat{\Gamma}_{\widehat{\alpha}}, \widehat{\mu}\right)$ is a saddle point for problem (3.1)-(3.2). In general, this saddle point is not unique, because $\widehat{\mu}(\cdot)$ can be any such that $R_{\widehat{\mu}}=R_{\text {min }}$.

Corollary 4.1 Consider two problems (3.1)-(3.2) such that the corresponding $(\mathcal{T}, M(\cdot)) \triangleq\left(\mathcal{T}^{(i)}, M^{(i)}(\cdot)\right)$ are different for $i=1,2$, but the corresponding $n$, $U, X_{0}, T, \mathbf{D}$, and $R_{\text {min }}$ are the same. Then these two problems have the same optimal $H$-strategies.

\subsection{On calculation of the optimal strategy}

Note that equation (4.1) is the heat equation and has the fundamental solution

$$
p(y, \tau, x, t, R)=\frac{1}{\sqrt{2 \pi(\tau-t) R / T}} \exp \frac{-(y-x)^{2}}{2(\tau-t) R / T},
$$

and the solution can be expressed explicitly as

$$
u(x, t, R, \lambda)=\int_{-\infty}^{+\infty} p(y, T, x, t, R) \widehat{F}(y, R, \lambda) d y .
$$

Furthermore,

$$
\begin{aligned}
& \frac{\partial u}{\partial x}(x, t, R, \lambda) \\
& =\int_{-\infty}^{+\infty} \frac{\partial p}{\partial x}(y, T, x, t, R) \widehat{F}(y, R, \lambda) d y=\int_{-\infty}^{+\infty} \frac{y-x}{(T-t) R / T} p(y, T, x, t, R) \widehat{F}(y, R, \lambda) d y \\
& =\frac{T}{(T-t) R} \int_{-\infty}^{+\infty} y p(y, T, x, t, R) \widehat{F}(y, R, \lambda) d y-\frac{x T}{(T-t) R} u(x, R, \lambda) .
\end{aligned}
$$


If $\partial \widehat{F} / \partial y$ exists and the corresponding integrals converge, then

$$
\begin{aligned}
& \frac{\partial u}{\partial x}(x, t, R, \lambda)=\int_{-\infty}^{+\infty} \frac{\partial p}{\partial x}(y, T, x, t, R) \widehat{F}(y, R, \lambda) d y \\
& =-\int_{-\infty}^{+\infty} \frac{\partial p}{\partial y}(y, T, x, t, R) \widehat{F}(y, R, \lambda) d y=\int_{-\infty}^{+\infty} p(y, T, x, t, R) \frac{\partial \widehat{F}}{\partial y}(y, R, \lambda) d y .
\end{aligned}
$$

Corollary 4.2 The optimal strategy can be presented as

$$
\widehat{\pi}(t)^{\top}=\frac{T}{(T-t) R_{\text {min }}}\left[\mathbf{E}_{*}\left\{Y(T, \mu(\cdot)) \widehat{F}\left(Y(T, \mu(\cdot)), R_{\text {min }}, \widehat{\lambda}_{\widehat{\mu}}\right) \mid \mathcal{F}_{t}^{\mu}\right\}-\widetilde{X}(t)\right] \widetilde{a}(t) Q(t)^{\top},
$$

where $\mathcal{F}_{t}^{\mu}$ is the filtration generated by $(S(t), \mu(t))$. If $\partial \widehat{F} / \partial y$ exists and the corresponding integrals in (4.8) converges, then

$$
\widehat{\pi}(t)^{\top}=\mathbf{E}_{*}\left\{\frac{\partial \widehat{F}}{\partial y}\left(Y(T, \mu(\cdot)), R_{\text {min }}, \widehat{\lambda}_{\widehat{\mu}}\right) \mid \mathcal{F}_{t}^{\mu}\right\} \widetilde{a}(t) Q(t)^{\top} .
$$

\section{Some examples}

Clearly, our maximin setting does not make sense for the problem with observable $\mu(t)$, if, for example, $\mu(t) \equiv \Theta$, where $\Theta$ is a random vector that is constant in time. In that case, one can identify $\Theta$ instantly. Another example when the maximin setting is meaningless is a problem when the optimal strategy is myopic. In this section, we give some examples of different models to show that our problem setting is meaningful for the case when optimal strategies are non-myopic. We discuss also what happen if we reduce the class of admissible strategies assuming that the strategies can use only observations of $(S, r)$.

\subsection{Myopic and non-myopic optimal strategies}

Proposition 5.1 Let $X_{0}=X(0)>0$. Further, let $M(t, \alpha, \xi) \equiv M(t, \alpha)$ does not depend on $\xi \in C\left([0, T] ; \mathbf{R}^{n}\right)$, and let at least one of the following conditions be satisfied:

(i) $U(x)=\log (x)$ and $(0,+\infty) \subset \mathbf{D}$;

(ii) $U(x)=x^{\delta}$ and $(0,+\infty) \subset \mathbf{D}$; where $\delta<1, \delta \neq 0$;

(iii) $U(x)=-k x^{2}+c x$ and $\mathbf{D}=\mathbf{R}$, where $k \in \mathbf{R}$ and $c \geq 0$.

Then there exists $C_{0}, C_{1}, \nu \in \mathbf{R}$ such that $C_{1} \neq 0, \nu \neq 0$ are constants, and that the optimal strategy $\pi(\cdot) \in \widetilde{\Sigma}\left(\mathcal{F}^{\mu}\right)$ for the problem (3.1)-(3.2) has the form

$$
\pi(t)^{\top}=\nu B(t)\left(\widetilde{X}(t)-C_{0}\right) \widetilde{a}(t)^{\top} Q(t),
$$


where $\tilde{X}(t)$ is the corresponding normalized wealth, $Q(t) \triangleq\left(\sigma(t) \sigma(t)^{\top}\right)^{-1}$. This solution is optimal for the problem

$$
\text { Maximize } \quad \mathbf{E} U(\tilde{X}(T, \Gamma(\cdot), \mu(\cdot))) \quad \text { over } \quad \Gamma(\cdot)
$$

for any $\mu(\cdot)$. If condition (i) is satisfied, then $\nu=1, C_{0}=0$. If condition (ii) is satisfied, then $\nu=1 /(1-\delta), C_{0}=0$.

It follows from this Proposition 5.1 that the optimal strategies are myopic for the case when either condition (i) or condition (ii) is satisfied, since the optimal strategy can be presented as $\pi(t)=\Gamma\left(t,[S(\cdot), \mu(\cdot)]_{[0, t]}\right)$ for all $(\mu(\cdot), T)$, where $\Gamma(\cdot)$ is a $H$-strategy, and this function $\Gamma(\cdot)$ does not depend on $(\mu(\cdot), T)$. In that case, our maximin setting does not make sense, since the optimal strategy can be obtained from observations of historical $\mu(t)$ and $S(t)$ without knowledge of their future distributions.

However, the optimal solution for a general case uses information about distribution of future values of $\mu(\cdot)$, and our maximin setting is meaningful. For instance, if condition (iii) of Proposition 5.1 is satisfied, then $C_{0}$ depends on $(\mu(\cdot), T)$, the optimal strategy is non-myopic, and the maximin setting makes sense. This can be also illustrated with one more example.

Example 5.1 Consider a model when the appreciation rate is random and constant. Let $n=1$, let $r$, and $\widetilde{a}$ be given constants, and let $\Delta=\left[\sigma_{0}, \sigma_{1}\right] \subset \mathbf{R}, \mathcal{T}=\left[\alpha_{0}, \alpha_{1}\right] \subset \mathbf{R}$ be given, where $\sigma_{0}<\sigma_{1}$ and $\alpha_{0}<\alpha_{1}$. Let $v: C([0, T / 2]) \times \mathcal{T} \rightarrow \Delta$ be a given measurable mapping. Let

$$
(r(t), \widetilde{a}(t)) \equiv(r, \widetilde{a}), \quad \sigma(t)=\left\{\begin{array}{cc}
\sigma_{1}, & t<T / 2 \\
\eta, & t \geq T / 2
\end{array}\right.
$$

where $r, \widetilde{a}$ are constants, $\eta \triangleq v\left(\left.S(\cdot)\right|_{[0, T / 2]}, \Theta\right)$, and where $\Theta$ is a random variable that does not depend on $w(\cdot)$ and can take values in $\mathcal{T}$. This is a special case of our model with

$$
\left(\bar{M}_{r}(t, \alpha), \bar{M}_{a}(t, \alpha)\right) \equiv(r, \widetilde{a}), \quad \bar{M}_{\sigma}(t, \alpha)= \begin{cases}\sigma_{1}, & t<T / 2 \\ v\left(\left.S(\cdot)\right|_{[0, T / 2]}, \alpha\right), & t \geq T / 2\end{cases}
$$

We assume that $v(\cdot)$ is such that $\bar{M}_{\sigma}$ satisfies conditions of regularity imposed in Section 2.3. In addition, we assume that $v\left(\xi, \alpha_{i}\right)=\sigma_{i}$ for all $\xi \in C([0, T / 2]), i=0,1$. 
Let $\kappa \in[0,1)$ and a random $\Theta$ be given, then $\mu(\cdot) \in \mathcal{A}(\mathcal{T})$ is uniquely defined. Consider the problem

$$
\begin{array}{ll}
\text { Maximize } & \mathbf{E} \log \tilde{X}(T, \Gamma(\cdot), \mu(\cdot)) \quad \text { over } \quad \Gamma(\cdot) \\
\text { subject to } & \left\{\begin{array}{l}
X(0, \Gamma(\cdot), \mu(\cdot))=X_{0}, \\
\tilde{X}(T, \Gamma(\cdot), \mu(\cdot)) \geq \kappa X_{0} \quad \text { a.s. }
\end{array}\right.
\end{array}
$$

By Lemma 4.1 (or by Theorem 5.1 (ii) from Dokuchaev and Haussmann (2001)) it follows that

- if either $\Theta \equiv \alpha_{0}$ or $\Theta \equiv \alpha_{1}$, then the optimal strategy exists; and

- if $\kappa \neq 0$, then the corresponding optimal strategies for these two cases differs at the time interval $[0, T / 2)$.

Hence the optimal strategy for problem (5.3) cannot be obtained from observations of historical $(r(t), \widetilde{a}(t), \sigma(t), S(t))$ without knowledge of future distributions. The only exception is the case $\kappa=0$, when the optimal strategy given $\mu(\cdot)$ is myopic.

Assume that $\kappa>0$. Then our solution in maximin setting for corresponding problem (3.1)-(3.2) is given in Lemma 4.1 with constant $\widehat{\mu}=(r, \widetilde{a}, \widehat{\sigma})$, where $\widehat{\sigma}=\sigma_{1}$. As far as we know, the existing methods described in literature does not allow to solve problem (5.3) for a given pair $(\Theta, v(\cdot))$ for a non-trivial case.

\subsection{Saddle point when the market parameters are non-observable}

Let $\mathcal{C}_{\text {hist }}$ be the class of admissible $H$-strategies $\Gamma(\cdot) \in \mathcal{C}$ such that the corresponding selffinancing strategy $\pi(t)$ is adapted to the filtration generated by $(S(t), r(t))$. In that case, the appreciation rates $\widetilde{a}(t)$ are not available for observations, and the strategy is based on historical prices only, i.e., $\pi(t)=\Gamma\left(t,\left.[S(\cdot), r(\cdot)]\right|_{[0, t]}\right)$, where $\Gamma(\cdot) \in \mathcal{C}_{\text {hist }}$ is a measurable function, $\Gamma(t, \cdot): B\left([0, t] ; \mathbf{R}^{n} \times \mathbf{R}\right) \rightarrow \mathbf{R}^{n}, t \geq 0$.

Note that if the process $\sigma(\cdot)$ is deterministic, then this setting can be reduced to the problem with observed parameters. The equation for $S$ can replaced for a similar equation, where $a(t)$ is replaced by $\widehat{a}(t) \triangleq \mathbf{E}\left\{a(t)|S(\cdot)|_{[0, t]}\right\}$, and where $w(t)$ is replaced by some new Brownian motion.

The following example was obtained numerically in Dokuchaev and Teo (1999). It shows that, for the maximin problem stated for the class $\mathcal{C}_{\text {hist }}$, the "worst" $\mu=\widehat{\mu}$ can be 
such that

$$
\mathbf{P}\left(\int_{0}^{T}\left|\theta_{\widehat{\mu}}(t)\right|^{2} d t>R_{\text {min }}\right)>0
$$

Example 5.2 Consider a model when the appreciation rate is random and constant. Let $n=1$, let $\alpha_{1}, \alpha_{2}$ and $\sigma$ be given constants, and let $\mathcal{T}=\left\{\alpha_{1}, \alpha_{2}\right\} \subset \mathbf{R}$. Assume that the set of processes $\mu(t)=(r(t), \widetilde{a}(t), \sigma(t))$ consists of all processes such that

$$
r(t) \equiv 0, \quad \sigma(t) \equiv \sigma, \quad \widetilde{a}(t) \equiv \Theta,
$$

where $\Theta$ is a random vector independent of $w(\cdot)$ and such that $\Theta \in \mathcal{T}$ a.s. This model can be described as a special case of our model with $\bar{E}=\mathbf{R}$, and

$$
\left(\bar{M}_{r}(t, \alpha), \bar{M}_{\sigma}(t, \alpha)\right) \equiv(0, \sigma), \quad \bar{M}_{a}(t, \alpha) \equiv \alpha, \quad \alpha \in \mathcal{T}=\left\{\alpha_{1}, \alpha_{2}\right\}
$$

Consider the optimal investment problem:

$$
\begin{array}{llrl}
\text { Maximize } & \min _{\mu(\cdot)} \mathbf{E} \ln \tilde{X}(T, \Gamma(\cdot), \mu(\cdot)) \quad \text { over } \quad \Gamma(\cdot) \in \mathcal{C}_{\text {hist }} \\
\text { subject to } & 0.95 \cdot X_{0} \leq X(T, \Gamma(\cdot), \mu(\cdot)) \leq 1.1 \cdot X_{0} .
\end{array}
$$

This problem is a special case of the problem (3.1)-(3.2) stated for the class of admissible strategies $\mathcal{C}_{\text {hist }}$. Dokuchaev and Teo (1999) presented a numerical solution with the following parameters: $S(0)=1.6487, X_{0}=1, T=1, \sigma=0.5, \alpha_{1}=0.2, \alpha_{2}=\log \left(2-e^{0.2}\right)=$ -0.2503. (With this parameters, $\mathbf{E} S(T)=S(0)$ if $\mathbf{P}\left(\widetilde{a}(t) \equiv \alpha_{1}\right)=\mathbf{P}\left(\widetilde{a}(t) \equiv \alpha_{1}\right)=1 / 2$.) A saddle point $(\widehat{\Gamma}(\cdot), \widehat{\mu}(\cdot))$ was found, where $\widehat{\Gamma}(\cdot)$ is the optimal strategy in the class $\mathcal{C}_{\text {hist }}$ and where $\widehat{\mu}(\cdot)$ is the "worst" market parameter. The result was that for the "worst" $\widehat{\mu}(\cdot)=(0, \widetilde{a}(\cdot), \sigma)$ we have

$$
\mathbf{P}\left(\widetilde{a}(t) \equiv \alpha^{(1)}\right)=0.5445, \quad \mathbf{P}\left(\widetilde{a}(t) \equiv \alpha^{(2)}\right)=0.4554 .
$$

Therefore, the worst parameter is pure stochastic here. It follows from this example that the duality theorem does not hold, if the class of random vectors $\Theta$ in the definition of the class of admissible $\mu(\cdot)$ is replaced by a class of deterministic vectors $\{\alpha\}$.

It was calculated also that $\mathbf{E} \ln \tilde{X}(T, \widehat{\Gamma}(\cdot), \widehat{\mu}(\cdot))=0.004$.

Let us discuss Example 5.2 in connection with the main result of the present paper. Clearly, the minimum of $R_{\mu}=\int_{0}^{T}\left|\theta_{\mu}(t)\right|^{2} d t$ is achieved for $\widetilde{a} \equiv \alpha_{1}$; for any other random $\widetilde{a}$ the value of $R_{\mu}$ is higher. Therefore, we have inequality (5.4). In fact, it is a consequence 
of the fact that $\alpha_{1} \alpha_{2}<0$ and therefore one can find $\mu$ such that $\int_{0}^{T}\left|\widehat{a}_{\mu}(t)\right|^{2} d t<\int_{0}^{T} \alpha_{1}^{2} d t$ a.s., where $\widehat{a}_{\mu}(t) \triangleq \mathbf{E}\left\{\Theta|S(\cdot)|_{[0, t]}\right\}$ for $\Theta$ that corresponds $\mu$. Thus, Condition 3.5 is not satisfied if one reformulates this problem as a problem with observable parameters with $a(t)$ replaced by the observable $\widehat{a}_{\mu}(t)$ and with $w(t)$ replaced by a new Wiener process obtained from the equation for the filter.

\section{Appendix: Proofs}

Proof of Lemma 4.1 The statement (i) is obvious. By the definition of $\widehat{F}$, we have that $\widehat{F}\left(Y\left(T, \mu_{\alpha}(\cdot)\right), R_{\mu_{\alpha}}, \widehat{\lambda}_{\alpha}\right)=F\left(\mathcal{Z}\left(T, \mu_{\alpha}(\cdot)\right), \widehat{\lambda}_{\alpha}\right)$. Set

$$
\widetilde{x}(t) \triangleq u\left(Y\left(t, \mu_{\alpha}(\cdot)\right), \tau_{\mu_{\alpha}}\left(t, R_{\mu_{\alpha}}\right), R_{\mu_{\alpha}}, \widehat{\lambda}_{\alpha}\right)
$$

By (4.1) and Itô's formula,

$$
\widetilde{x}(T)=\widehat{F}\left(Y\left(T, \mu_{\alpha}(\cdot)\right), R_{\mu_{\alpha}}, \widehat{\lambda}_{\alpha}\right)=F\left(\mathcal{Z}\left(T, \mu_{\alpha}(\cdot)\right), \widehat{\lambda}_{\alpha}\right),
$$

and

$$
\widetilde{x}(T)=\widetilde{x}(0)+\int_{0}^{T} B(t)^{-1} \widehat{\pi}_{\alpha}(t)^{\top} \sigma(t) d w_{*}(t)=\widetilde{x}(0)+\int_{0}^{T} \widehat{\pi}_{\alpha}(t)^{\top} \mathbf{S}(t)^{-1} d \widetilde{S}(t) .
$$

Here $w_{*}(t) \triangleq w(t)+\int_{0}^{t} \theta_{\mu_{\alpha}}(s) d s$. Girsanov Theorem implies that $w_{*}$ is the standard Wiener process under $\mathbf{P}_{*}^{\alpha}$. By Condition 3.4, $\widetilde{x}(0)=X_{0}$. By $(2.5), \widetilde{x}(t)$ is the normalized wealth that corresponds to $\widehat{\pi}_{\alpha}(\cdot)$. Then (4.3) follows for $\tilde{X}(t) \equiv \widetilde{x}(t)$. Then statement (iii) and optimality of the strategy follows from Theorem 5.1, from Dokuchaev and Haussmann (2001).

\section{A.1 Additional definitions}

Without loss of generality, we describe the probability space as follows: $\Omega=\mathcal{T} \times \Omega^{\prime}$, where $\Omega^{\prime}=C\left([0, T] ; \mathbf{R}^{n}\right)$. We are given a $\sigma$-algebra $\mathcal{F}^{\prime}$ of subsets of $\Omega^{\prime}$ generated by cylindrical sets, and a $\sigma$-additive probability measure $\mathbf{P}^{\prime}$ on $\mathcal{F}^{\prime}$ generated by $w(\cdot)$. Furthermore, let $\mathcal{F}_{\mathcal{T}}$ be the $\sigma$-algebra of all Borel subsets of $\mathcal{T}$, and $\mathcal{F}=\mathcal{F}_{\mathcal{T}} \otimes \mathcal{F}^{\prime}$. We assume also that each $\mu(\cdot) \in \mathcal{A}(\mathcal{T})$ generates the $\sigma$-additive probability measure $\nu_{\mu}$ on $\mathcal{F}_{\mathcal{T}}$ (this measure is generated by $\Theta$ which corresponds to $\mu(\cdot))$.

Let $\stackrel{\circ}{\mathbf{R}}_{+}^{n} \triangleq(0,+\infty)^{n}$. 
For a function $\Gamma(t, \cdot): C\left([0, t] ; \stackrel{\circ}{\mathbf{R}}_{+}^{n}\right) \times B\left([0, t] ; \mathbf{R} \times \mathbf{R}^{n} \times \mathbf{R}^{n \times n}\right) \rightarrow \mathbf{R}^{n}$, introduce the following norm:

$$
\|\Gamma(\cdot)\|_{\mathbf{X}} \triangleq \sup _{\mu(\cdot)=\mu_{\alpha}(\cdot): \alpha \in \mathcal{T}}\left(\mathbf{E} \sum_{i=1}^{n} \int_{0}^{T} \Gamma_{i}\left(t,\left.[S(\cdot), \mu(\cdot)]\right|_{[0, t]}\right)^{2} d t\right)^{1 / 2} .
$$

Definition A.1 Let $\mathcal{C}_{0}$ be the set of all admissible $H$-strategies $\Gamma(t, \cdot): C\left([0, t] ; \mathbf{R}^{n}\right) \times$ $B\left([0, t] ; \mathbf{R} \times \mathbf{R}^{n} \times \mathbf{R}^{n \times n}\right) \rightarrow \mathbf{R}^{n}$ such that

$$
\begin{aligned}
& \|\Gamma(\cdot)\|_{\mathbf{X}}<+\infty, \\
& \pi(t)=\Gamma\left(t,\left.[S(\cdot), \mu(\cdot)]\right|_{[0, t]}\right) \in \widetilde{\Sigma}\left(\mathcal{F}^{\mu}\right) \quad \forall \mu(\cdot) \in \mathcal{A}(\mathcal{T}), \\
& \widetilde{X}(T, \Gamma(\cdot), \mu(\cdot)) \in \mathbf{D} \quad \text { a.s. } \quad \forall \mu(\cdot) \in \mathcal{A}(\mathcal{T}) .
\end{aligned}
$$

In fact, $\mathcal{C}_{0}$ is a subset of the linear space of functions with finite norm (A.1).

\section{A.2 A duality theorem}

To prove Theorem 4.1, we need the following duality theorem.

Theorem A.1 The following holds:

$$
\begin{aligned}
\sup _{\Gamma(\cdot) \in \mathcal{C}_{0}} & \inf _{\mu \in \mathcal{A}(\mathcal{T})} \mathbf{E} U(\tilde{X}(T, \Gamma(\cdot), \mu(\cdot))) \\
& =\inf _{\mu(\cdot) \in \mathcal{A}(\mathcal{T})} \sup _{\Gamma(\cdot) \in \mathcal{C}_{0}} \mathbf{E} U(\widetilde{X}(T, \Gamma(\cdot), \mu(\cdot))) .
\end{aligned}
$$

By this theorem, it follows that there exists a saddle point, i.e., a common solution $(\Gamma(\cdot), \mu(\cdot))$ for the maximin problem and for the minimax problem.

To prove Theorem A.1, we need several preliminary results, which are presented below as lemmas.

Lemma A.1 The function $\tilde{X}(T, \Gamma(\cdot), \mu(\cdot))$ is affine in $\Gamma(\cdot)$.

Proof. By $(2.5)$, it follows that $\tilde{X}(t)=\tilde{X}(t, \Gamma(\cdot), \mu(\cdot))$ satisfies

$$
\begin{aligned}
& \tilde{X}(t)=X(0) \\
& +\sum_{i=1}^{n} \int_{0}^{t} B(\tau)^{-1} \Gamma_{i}\left(\tau,\left.[S(\cdot, \mu(\cdot)), \mu(\cdot)]\right|_{[0, \tau]}\right)\left[\widetilde{a}_{i}(\tau) d \tau+\sum_{j=1}^{n} \sigma_{i j}(\tau) d w_{j}(\tau)\right] .
\end{aligned}
$$

It is easy to see that $\widetilde{X}(T, \Gamma(\cdot), \mu(\cdot))$ is affine in $\Gamma(\cdot)$. This completes the proof.

Lemma A.2 The set $\mathcal{C}_{0}$ is convex. 
Proof. Let $p \in(0,1), \mu(\cdot) \in \mathcal{A}(\mathcal{T}), \Gamma^{(i)}(\cdot) \in \mathcal{C}_{0}, i=1,2$, and let

$$
\Gamma(\cdot) \triangleq(1-p) \Gamma^{(1)}(t)+p \Gamma^{(2)}(\cdot)
$$

By Lemma A.1, it follows that

$$
\widetilde{X}(T, \Gamma(\cdot), \mu(\cdot))=(1-p) \widetilde{X}\left(T, \Gamma^{(1)}(\cdot), \mu(\cdot)\right)+p \widetilde{X}\left(T, \Gamma^{(2)}(\cdot), \mu(\cdot)\right) .
$$

The set $\mathbf{D}$ is convex, then $\widetilde{X}(t, \Gamma(\cdot), \mu(\cdot)) \in \mathbf{D}$ a.s.. This completes the proof.

Lemma A.3 There exists a constant $c>0$ such that

$$
\mathbf{E}\left|\widetilde{X}\left(T, \Gamma(\cdot), \mu_{\alpha}(\cdot)\right)\right|^{2} \leq c\left(\|\Gamma(\cdot)\|_{\mathbf{X}}^{2}+X_{0}^{2}\right) \quad \forall \Gamma(\cdot) \in \mathcal{C}_{0}, \quad \forall \alpha \in \mathcal{T}
$$

Proof follows from the properties of the stochastic integrals and from (A.3) applied for $\mu=\mu_{\alpha}$.

Lemma A.4 The function

$$
\mathbf{E} U\left(\widetilde{X}\left(T, \Gamma(\cdot), \mu_{\alpha}(\cdot)\right)\right)
$$

is continuous in $\Gamma(\cdot) \in \mathcal{C}_{0}$ uniformly in $\alpha \in \mathcal{T}$.

Proof. Let $\Gamma^{(i)}(\cdot) \in \mathcal{C}_{0}$ and $\widetilde{X}^{(i)}(t) \triangleq \widetilde{X}\left(t, \Gamma^{(i)}(\cdot), \mu_{\alpha}(\cdot)\right), i=1,2$. By Lemmas A.1 and A.3, it follows that

$$
\mathbf{E}\left|\widetilde{X}^{(1)}(T)-\widetilde{X}^{(2)}(T)\right|^{2} \leq c\left\|\Gamma^{(1)}(\cdot)-\Gamma^{(2)}(\cdot)\right\|_{\mathbf{X}}^{2},
$$

where $c>0$ is a constant. Then

$$
\begin{aligned}
\mid \mathbf{E} U\left(\widetilde{X}^{(1)}(T)\right) & -\mathbf{E} U\left(\widetilde{X}^{(2)}(T)\right) \mid \\
& \leq c_{1} \mathbf{E}\left[\left(1+\left|\widetilde{X}^{(1)}(T)\right|+\left|\widetilde{X}^{(2)}(T)\right|\right)^{2-q}\left|\widetilde{X}^{(1)}(T)-\widetilde{X}^{(2)}(T)\right|^{q}\right] \\
& \leq c_{1}\left[\mathbf{E}\left(1+\left|\widetilde{X}^{(1)}(T)\right|+\left|\widetilde{X}^{(2)}(T)\right|\right)^{2}\right]^{1 / k^{\prime}}\left[\mathbf{E}\left|\widetilde{X}^{(1)}(T)-\widetilde{X}^{(2)}(T)\right|^{2}\right]^{1 / k} \\
& \leq c_{2}\left(1+\left\|\Gamma^{(1)}(\cdot)\right\|_{\mathbf{X}}^{2}+\left\|\Gamma^{(2)}(\cdot)\right\|_{\mathbf{X}}^{2}\right)^{1 / k^{\prime}}\left\|\Gamma^{(1)}(\cdot)-\Gamma^{(2)}(\cdot)\right\|_{\mathbf{X}}^{2 / k},
\end{aligned}
$$

where $q$ is as defined in Condition 3.1, $k \triangleq 2 / q, k^{\prime} \triangleq k /(k-1)=2 /(2-q)$ and $c_{i}>0$ are constants that do not depend on $\alpha$. This completes the proof.

For $\alpha \in \mathcal{T}$, set

$$
J^{\prime}(\Gamma(\cdot), \alpha) \triangleq \mathbf{E} U\left(\tilde{X}\left(T, \Gamma(\cdot), \mu_{\alpha}(\cdot)\right)\right)
$$


Lemma A.5 For a given $\Gamma(\cdot) \in \mathcal{C}_{0}$, the function $J^{\prime}(\Gamma(\cdot), \alpha)$ is continuous in $\alpha \in \mathcal{T}$.

Proof. Let $\mathcal{C}_{\text {Lip }}$ denote the class of functions $\Gamma(\cdot) \in \mathcal{C}_{0}$ such that there exists a constant $C=C(\Gamma(\cdot))>0$ such that

$$
\begin{aligned}
& \left.\sup _{t \in[0, T]}|\Gamma(t, x(\cdot))|_{[0, t]}\right)-\Gamma\left(t,\left.y(\cdot)\right|_{[0, t]}\right)\left|\leq C \sup _{t \in[0, T]}\right| x(t)-y(t) \mid, \\
& \left.\sup _{t \in[0, T]}|\Gamma(t, x(\cdot))|_{[0, t]}\right) \mid \leq C \sup _{t \in[0, T]}(|x(t)|+1) \\
& \forall x, y \in C\left([0, T] ; \mathbf{R}^{n}\right) \times B\left([0, T] ; \mathbf{R} \times \mathbf{R}^{n} \times \mathbf{R}^{n \times n}\right) .
\end{aligned}
$$

Clearly, the set $\mathcal{C}_{\text {Lip }}$ is elsewhere dense in $\mathcal{C}_{0}$ provided with the norm $\|\cdot\| \mathbf{X}$.

It suffices to show that, for any $\widetilde{\alpha} \in \mathcal{A}(\mathcal{T})$ and $\varepsilon>0$, there exists $\delta>0$ such that if $|\alpha-\widetilde{\alpha}| \leq \delta$ then $\left|J^{\prime}(\Gamma(\cdot), \alpha)-J^{\prime}(\Gamma(\cdot), \widetilde{\alpha})\right| \leq \varepsilon$.

By Lemma A.4, the function $J^{\prime}(\Gamma(\cdot), \alpha)$ is continuous in $\Gamma \in \mathcal{C}_{0}$ uniformly in $\alpha \in \mathcal{T}$. Let $\Gamma^{\prime}(\cdot) \in \mathcal{C}_{\text {Lip }}$ be such that $\left|J^{\prime}(\Gamma(\cdot), \alpha)-J^{\prime}\left(\Gamma^{\prime}(\cdot), \alpha\right)\right| \leq \varepsilon / 3$ for all $\alpha$.

By $(2.2)$ and (A.3), the process $\zeta(t, \alpha) \triangleq\left(\widetilde{X}\left(t, \Gamma^{\prime}(\cdot), \mu_{\alpha}(\cdot)\right), S\left(t, \mu_{\alpha}(\cdot)\right)\right.$ is the solution of an Itô's equation with smooth enough coefficients that continuously depend on $\alpha$ such that conditions of Theorem 3.15 from Gikhman and Skorohod (1979) are satisfied. By this theorem, $\mathbf{E} \sup _{t \in[0, T]}\left|\zeta\left(t, \alpha_{1}\right)-\zeta\left(t, \alpha_{2}\right)\right|^{2} \rightarrow 0$ as $\alpha_{1} \rightarrow \alpha_{2}$. Hence $J^{\prime}\left(\Gamma^{\prime}(\cdot), \alpha\right)$ is continuous in $\alpha \in A$. (There are many other available results about dependence on parameters for solutions of Itô's equations; see, e.g., Chapter 2 in Krylov (1980)). Let $\delta>0$ be such that $\left|J^{\prime}\left(\Gamma^{\prime}(\cdot), \alpha\right)-J^{\prime}\left(\Gamma^{\prime}(\cdot), \widetilde{\alpha}\right)\right| \leq \varepsilon / 3$ for all $\alpha$ such that $|\alpha-\widetilde{\alpha}|<\delta$.

We have that

$$
\begin{aligned}
& \left|J^{\prime}(\Gamma(\cdot), \alpha)-J^{\prime}(\Gamma(\cdot), \widetilde{\alpha})\right| \leq\left|J^{\prime}(\Gamma(\cdot), \alpha)-J^{\prime}\left(\Gamma^{\prime}(\cdot), \alpha\right)\right| \\
& \quad+\left|J^{\prime}\left(\Gamma^{\prime}(\cdot), \alpha\right)-J^{\prime}\left(\Gamma^{\prime}(\cdot), \widetilde{\alpha}\right)\right|+\left|J^{\prime}\left(\Gamma^{\prime}(\cdot), \widetilde{\alpha}\right)-J^{\prime}(\Gamma(\cdot), \widetilde{\alpha})\right| \leq \varepsilon .
\end{aligned}
$$

Thus, $\delta$ is such as required. This completes the proof.

Let $\mathcal{V}$ be the set of all $\sigma$-additive probability measures on $\mathcal{F}_{\mathcal{T}}$. We consider $\mathcal{V}$ as a subset of $C(\mathcal{T} ; \mathbf{R})^{*}$. Let $\mathcal{V}$ be equipped with the weak* topology in the sense that

$$
\nu_{1} \rightarrow \nu_{2} \quad \Leftrightarrow \quad \int_{\mathcal{T}} \nu_{1}(d \alpha) f(\alpha) \rightarrow \int_{\mathcal{T}} \nu_{2}(d \alpha) f(\alpha) \quad \forall f(\cdot) \in C(\mathcal{T} ; \mathbf{R}) .
$$

Lemma A.6 The set $\mathcal{V}$ is compact and convex.

Proof. The convexity is obvious. It remains to show the compactness of the set $\mathcal{V}$. In our case, the set $\mathcal{T}$ is a compact subset of a finite-dimensional Euclidean space. Now we note that the Borel $\sigma$-algebra of subsets of $\mathcal{T}$ coincides with the Baire $\sigma$-algebra (see, 
e.g., Bauer (1981)). Hence, $\mathcal{V}$ is the set of Baire probability measures. By Theorem IV.1.4 from Warga (1972), it follows that $\mathcal{V}$ is compact. This completes the proof.

We are now in the position to give a proof of Theorem A.1.

Proof of Theorem A.1. For a $\Gamma(\cdot) \in \mathcal{C}_{0}$, we have $J^{\prime}(\Gamma(\cdot), \cdot) \in C(\mathcal{T} ; \mathbf{R})$ and

$$
\begin{aligned}
\mathbf{E} U(\tilde{X}(T, \Gamma(\cdot), \mu(\cdot))) & =\int_{\mathcal{T}} d \nu_{\mu}(\alpha) \mathbf{E} U\left(\widetilde{X}\left(T, \Gamma(\cdot), \mu_{\alpha}(\cdot)\right)\right) \\
& =\int_{\mathcal{T}} d \nu_{\mu}(\alpha) J^{\prime}(\Gamma(\cdot), \alpha),
\end{aligned}
$$

where $\nu_{\mu}(\cdot)$ is the measure on $\mathcal{T}$ generated by $\Theta$ which corresponds $\mu(\cdot)$. Hence, $\mathbf{E} U(\tilde{X}(T, \Gamma(\cdot), \mu(\cdot)))$ is uniquely defined by $\nu_{\mu}$. Let

$$
J\left(\Gamma(\cdot), \nu_{\mu}\right) \triangleq \mathbf{E} U(\widetilde{X}(T, \Gamma(\cdot), \mu(\cdot))) .
$$

By Lemma A.5, $J(\Gamma(\cdot), \nu)$ is linear and continuous in $\nu \in \mathcal{V}$ given $\Gamma(\cdot)$.

To complete the proof, it suffices to show that

$$
\sup _{\Gamma(\cdot) \in \mathcal{C}_{0}} \inf _{\nu \in \mathcal{V}} J(\Gamma(\cdot), \nu)=\inf _{\nu \in \mathcal{V}} \sup _{\Gamma(\cdot) \in \mathcal{C}_{0}} J(\Gamma(\cdot), \nu) .
$$

We note that $J(\Gamma(\cdot), \nu): \mathcal{C}_{0} \times \mathcal{V} \rightarrow \mathbf{R}$ is linear in $\nu$. By Lemmas A.1 and A.4-A.5, it follows that $J(\Gamma(\cdot), \nu)$ is either concave or convex in $\Gamma(\cdot)$ and that $J(\Gamma(\cdot), \nu): \mathcal{C}_{0} \times \mathcal{V} \rightarrow \mathbf{R}$ is continuous in $\nu$ for each $\Gamma(\cdot)$ and continuous in $\Gamma(\cdot)$ for each $\nu$. Furthermore, the sets $\mathcal{C}_{0}$ and $\mathcal{V}$ are both convex, and the set $\mathcal{V}$ is compact. By the Sion Theorem (see, e.g., Parthasarathy and Ragharan (1971, p.123)), it follows that (A.4), and hence (A.2), are satisfied. This completes the proof of Theorem A.1.

We are now in the position to give a proof of Theorem 4.1.

\section{A.3 Proof of Theorem 4.1}

Let $\widehat{\alpha} \in \mathcal{T}$ be such that $R_{\widehat{\mu}}=R_{\text {min }}$, where $\widehat{\mu}(\cdot) \triangleq \mu_{\widehat{\alpha}}(\cdot)$. Let $\lambda=\widehat{\lambda}$ be such as defined in Condition 3.3 for $\alpha=\widehat{a}$. By Lemma 4.1(ii), it follows that

$$
\mathbf{E} U\left(\widetilde{X}\left(T, \widehat{\Gamma}_{\widehat{\alpha}}(\cdot), \widehat{\mu}(\cdot)\right)\right)=\sup _{\Gamma(\cdot) \in \mathcal{C}_{0}} \mathbf{E} U(\widetilde{X}(T, \Gamma(\cdot), \widehat{\mu}(\cdot))) .
$$

Let $\mu(\cdot) \in \mathcal{A}(\mathcal{T})$ be arbitrary. Let

$$
\widetilde{T}_{\mu}(t) \triangleq \inf \left\{s: \int_{0}^{s}\left|\theta_{\mu}(r)\right|^{2} d r>t\right\}, \quad T_{\mu} \triangleq \widetilde{T}_{\mu}\left(R_{m i n}\right) .
$$

Clearly, $T_{\mu} \leq T$, and $T_{\mu}=\inf \left\{t: \tau_{\mu}\left(t, R_{\text {min }}\right)=T\right\}$, where $\tau_{\mu}(t, R) \triangleq \frac{T}{R} \int_{0}^{t}\left|\theta_{\mu}(s)\right|^{2} d s$. 
Set $I_{\mu}(t) \triangleq \int_{0}^{t} \theta_{\mu}(s)^{\top} d w(s)$. This is a martingale. By the Dambis-Dubins-Schwarz theorem, $I_{\mu}^{\prime}(t) \triangleq I_{\mu}\left(\widetilde{T}_{\mu}(t)\right)$ is a Brownian motion (see Revuz and Your (1999)). We have that

$$
\begin{array}{cc}
Y(T, \widehat{\mu}(\cdot))=\int_{0}^{T}\left|\theta_{\widehat{\mu}}(t)\right|^{\top} d w(s), & \ln \mathcal{Z}(T, \widehat{\mu}(\cdot))=\int_{0}^{T}\left|\theta_{\widehat{\mu}}(t)\right|^{\top} d w(s)+\frac{1}{2} R_{\text {min }}, \\
Y\left(T_{\mu}, \mu(\cdot)\right)=I_{\mu}\left(T_{\mu}\right)=I_{\mu}^{\prime}\left(R_{\text {min }}\right), & \ln \mathcal{Z}\left(T_{\mu}, \mu(\cdot)\right)=I_{\mu}\left(T_{\mu}\right)+\frac{1}{2} R_{\text {min }}=I_{\mu}^{\prime}\left(R_{\text {min }}\right)+\frac{1}{2} R_{\text {min }} .
\end{array}
$$

These two random variables are Gaussian with mean $R_{m i n} / 2$ and variance $R_{\text {min }}$. Therefore, the variables $Y(T, \widehat{\mu}(\cdot))$ and $Y\left(T_{\mu}, \mu(\cdot)\right)$ have the same probability distribution.

Let $u(\cdot)$ be such as defined in Lemma 4.1. It is easy to see that the process

$$
\tilde{X}^{\prime}(t)= \begin{cases}u\left(Y(t, \mu(\cdot)), \tau_{\mu}\left(t, R_{m i n}\right), R_{m i n}, \widehat{\lambda}\right), & t \leq T_{\mu} \\ \widetilde{X}^{\prime}\left(T_{\mu}\right), & t>T_{\mu}\end{cases}
$$

is the normalized self-financing wealth for some admissible strategy $\Gamma^{\prime}(\cdot) \in \mathcal{C}_{0}$, i.e., $\widetilde{X}^{\prime}(t)=$ $\widetilde{X}\left(t, \Gamma^{\prime}(\cdot), \mu(\cdot)\right)$. Furthermore,

$$
\tilde{X}^{\prime}(T)=\tilde{X}\left(T, \Gamma^{\prime}(\cdot), \mu(\cdot)\right)=F\left(\mathcal{Z}\left(T_{\mu}, \mu(\cdot)\right), \widehat{\lambda}\right)=\widehat{F}\left(Y\left(T_{\mu}, \mu(\cdot)\right), R_{m i n}, \widehat{\lambda}\right),
$$

and this variable has the same distribution as

$$
\widetilde{X}\left(T, \widehat{\Gamma}_{\widehat{\alpha}}(\cdot), \widehat{\mu}(\cdot)\right)=F(\mathcal{Z}(T, \widehat{\mu}(\cdot)), \widehat{\lambda})=\widehat{F}\left(Y(T, \widehat{\mu}(\cdot)), R_{\text {min }}, \widehat{\lambda}\right)
$$

Hence

$$
\mathbf{E} U\left(\widetilde{X}\left(T, \widehat{\Gamma}_{\widehat{\alpha}}(\cdot), \widehat{\mu}(\cdot)\right)\right)=\mathbf{E} U\left(\widetilde{X}\left(T, \Gamma^{\prime}(\cdot), \mu(\cdot)\right)\right)
$$

Therefore,

$$
\mathbf{E} U\left(\widetilde{X}\left(T, \widehat{\Gamma}_{\widehat{\alpha}}(\cdot), \widehat{\mu}(\cdot)\right)\right) \leq \sup _{\Gamma(\cdot) \in \mathcal{C}_{0}} \mathbf{E} U(\widetilde{X}(T, \Gamma(\cdot), \mu(\cdot))) \quad \forall \mu(\cdot) \in \mathcal{A}(\mathcal{T}) .
$$

By (A.5) and (A.6), the pair $\left(\widehat{\mu}(\cdot), \Gamma_{\widehat{\alpha}}(\cdot)\right)$ solves the problem

$$
\text { Minimize } \sup _{\Gamma(\cdot) \in \mathcal{C}_{0}} \mathbf{E} U(\tilde{X}(T, \Gamma(\cdot), \mu(\cdot))) \quad \text { over } \quad \mu(\cdot) \in \mathcal{A}(\mathcal{T}) .
$$

By Theorem A.1 it follows that the pair $\left(\widehat{\mu}(\cdot), \widehat{\Gamma}_{\widehat{\alpha}}(\cdot)\right)$ is a saddle point for problem (3.1)(3.2). This completes the proof of Theorem 4.1.

Proof of Remark 4.1 is obvious.

Proof of Corollary 4.1 follows from Lemma 4.1 (iii). 
Proof of Corollary 4.2. To prove (4.10), it suffices to note that $\frac{\partial u}{\partial x}$ satisfies the problem (4.1), where $\widehat{F}$ is replaced by $\frac{\partial \widehat{F}}{\partial x}$ in the Cauchy condition. Then the rest part of the proof is similar to the proof of (4.3) in Lemma 4.1. Proof of (4.9) is similar.

Proof of Proposition 5.1. By the assumptions on $M(\cdot)$, it follows that $\mu(\cdot) \in \mathcal{A}(\mathcal{T})$ does not depend on $w(\cdot)$. Condition 3.3 is satisfied with $F(x, \lambda)=C_{1}\left(\frac{x}{\lambda}\right)^{\nu}+C_{0}$, where $C_{1} \neq 0, C_{0}$ and $\nu \neq 0$ are constants. Then the proof follows from Corollary 5.1 from Dokuchaev and Haussmann (2001).

The first version of this paper was presented at the European Investment Review Conference, Paris, September 2001.

\section{References}

Ackermann, J. (1993) Robust control: systems with uncertain physical parameters. Springer-Verlag, Berlin.

Bauer, H. (1981): Probability Theory and Elements of Measure Theory. New York: Academic Press, 2nd ed.

Brennan, M.J. (1998): The role of learning in dynamic portfolio decisions. European Finance Rev. 1 (1998), 295-306.

Bryson, A., E., Jr. (1996) Optimal Control - 1950 to 1985. IEEE Control Systems 16 (3), 26-33.

Chacko, G., and Viceira, L.M. (2005): Dynamic consumption and portfolio choice with stochastic volatility in incomplete markets. Review of Financial Studies 18 (4), 1369-1402.

Cvitanić, J. (2000): Minimizing expected loss of hedging in incomplete and constrained markets. SIAM Journal of Control and Optimization 38, 1050-1066.

Cvitanić, J., and Karatzas, I. (1999): On dynamic measures of risk. Finance and Stochastics, 4, 451-482.

Cvitanic, J., Lazrak Ali, Martellini, L., Zapatero, F. (2005): Dynamic portfolio choice with parameter uncertainty and the economic value of analysts' recommendations. USC FBE Working Paper No. 02-8.

Detemple, J.B. (1986): Asset pricing in an economy with incomplete information. Journal of Finance 41, 369-382.

Dokuchaev, N.G., and Teo, K.L. (1999): A duality approach to an optimal investment problem with unknown and nonobservable parameters. Department of Applied Mathe- 
matics, Hong Kong Polytechnic University Working Paper.

Dokuchaev, N.G., and Haussmann, U. (2001): Optimal portfolio selection and compression in an incomplete market. Quantitative Finance, 1, 336-345.

Dokuchaev, N.G., and Zhou, X.Y. (2001): Optimal investment strategies with bounded risks, general utilities, and goal achieving. Journal of Mathematical Economics 35, iss. 2, 289-309.

Dothan, U., and Feldman, D. (1986): Equilibrium interest rates and multiperiod bonds in a partially observable economy. Journal of Finance 41, 369-382.

Epstein, L., and Chen, Z. (2002): Ambiguity, risk and asset returns in continuous time. Econometrica 70, 1403-1443.

Epstein, L., and Miao, J. (2003): A two-person dynamic equilibrium under ambiguity. Journal of Economic Dynamics and Control 27, 1253-1288.

Epstein, L., and Schneider, M. (2002): Learning Under Ambiguity/ RCER Working Papers 497, University of Rochester, Center for Economic Research (RCER).

Fleming, W.H., and Hernandez-Hernandez, D. (2003): An optimal consumption model with stochastic volatility. Finance and Stochastics 7, 245-262.

Fleming, W.H, and Pang, Tao. (2004): An application of stochastic control theory to financial economics. SIAM Journal on Control and Optimization 43, No 2, 502-531.

Föllmer, H., and Schied, A. (2002): Stochastic finance (Studies in Mathematics, Vol. 27). de Gruyter, Berlin.

Gennotte, G. (1986): Optimal portfolio choice under incomplete information. Journal of Finance 41 (1986), 733-749.

Gikhman, I.I., and Skorohod, A.V. (1979): Controlled stochastic processes. SpringerVerlag, New York-Heidelberg.

Gilboa, I. and Schmeidler, D. (1989): Maximin expected utility with non-unique prior. Journal of Mathematical Economics, 18, 141-153.

Hakansson, N. (1997): Portfolio analysis. W.W. Norton, New York.

Karatzas, I., and Shreve, S.E. (1998): Methods of mathematical finance. SpringerVerlag, New York.

Krylov, N.V. (1980): Controlled diffusion processes. Shpringer-Verlag.

Lakner, P. (1995): Utility maximization with partial information. Stochastic Processes Appl. 56, 247-273. 
Lakner, P. (1998): Optimal trading strategy for an investor: the case of partial information. Stoch. Processes Appl.. 76, 77-97.

Lazrak, Ali, and Zapatero, F. (2004): Efficient consumption set under recursive utility and unknown beliefs. Journal of Mathematical Economics 40, 207-226.

Merton, R. (1969): Lifetime portfolio selection under uncertainty: the continuoustime case. Rev. Econom. Statist. 51, 247-257.

Munk, C., and Sorensen, C. (2004): Optimal consumption and investment strategies with stochastic interest rates. Journal of Banking $\mathscr{E}$ Finance 28, iss. 8, 1987-2013.

Parthasarathy, T., and Raghavan, T.E.S. (1971): Some topics in two-person games. Elsevier, New York.

Revuz, M.J.D., and Yor, M. (1999). Continuous martingales and Brownian motion. Springer-Verlag, New York.

Sarr, J., and Haussmann, U. (2004): Optimizing the terminal wealth under partial information: the drift process as a continuous time Markov chain. Finance and Stochastics 8, 553-577.

Sircar, K. R., and Zariphopoulou, T. (2005): Bounds and asymptotic approximations for utility prices when volatility is random. SIAM Journal on Control Optimization 43, iss. $4,1328-1353$.

Trojany F, and Vanini P. (2002). Risk, robustness and Knightian Uncertainty in continuous-time, heterogenous agents, financial Equilibria (Working Paper, EFA 2002 Berlin Meetings).

Warga, J. (1972): Optimal control of differential and functional equations. Academic Press, New York.

Williams, J.T. (1977): Capital asset prices with heterogeneous beliefs. Journal of Financial Economics 5, 219-240. 